\title{
BMJ Open The effects of physical activity interventions on glycated haemoglobin A1c in non-diabetic populations: a protocol for a systematic review and meta-analysis
}

\author{
I Cavero-Redondo, ${ }^{1}$ B Peleteiro, ${ }^{2,3}$ C Álvarez-Bueno, ${ }^{1}$ M Garrido-Miguel, ${ }^{1}$ \\ E G Artero, ${ }^{4}$ V Martinez-Vizcaino ${ }^{1,5}$
}

To cite: Cavero-Redondo I, Peleteiro B, Álvarez-Bueno C, et al. The effects of physical activity interventions on glycated haemoglobin A1c in non-diabetic populations: a protocol for a systematic review and meta-analysis. BMJ Open 2017;7:e015801. doi:10.1136/ bmjopen-2016-015801

- Prepublication history for this paper is available online. To view these files please visit the journal online (http://dx.doi org/10.1136/bmjopen-2016015801).

Received 30 December 2016 Revised 26 April 2017 Accepted 22 May 2017

\section{CrossMark}

${ }^{1}$ Universidad de Castilla-La Mancha, Health and Social Research Center, Cuenca, Spain ${ }^{2}$ EPI Unit, Instituto de Saúde Pública, Universidade do Porto, Porto, Portugal

${ }^{3}$ Departamento de Ciências da Saúde Pública e Forenses e

Educação Médica, Faculdade de Medicina, Universidade do Porto, Porto, Portugal

${ }^{4}$ SPORT Research Group (CTS-1024), Area of Physical Education and Sport, University of Almería, Almería, Spain

${ }^{5}$ Universidad Autónoma de Chile, Facultad de Ciencias de la Salud, Talca, Chile

Correspondence to C Álvarez-Bueno; Celia. Alvarezbueno@uclm.es

\section{ABSTRACT}

Introduction Epidemiological evidence suggests that physical activity has a positive effect on reducing glycated haemoglobin A1c (HbA1c) levels not only in diabetics, but also in healthy subjects. Moreover, a positive association of $\mathrm{HbA} 1 \mathrm{c}$ levels with cardiovascular disease and mortality in non-diabetic populations has recently been reported. This is a protocol for a systematic review and metaanalysis aiming to estimate the effects of physical activity on glycaemic control measured by $\mathrm{HbA1c}$ levels in nondiabetic populations; and to determine which type of physical activity has a greater influence on glycaemic control.

Methods and analysis The search will be conducted using MEDLINE, EMBASE, the Cochrane Library and Web of Science databases from inception to mid2017. Randomised controlled trials, non-randomised experimental studies and controlled pre-post studies written in English, Portuguese, French or Spanish will be included. The Cochrane Collaboration's tool and The Quality Assessment Tool for Quantitative Studies will be used to assess the risk of bias for studies included in the systematic review. Standardised pre-post intervention mean differences of $\mathrm{HbA} 1 \mathrm{c}$ will be calculated as the primary outcome. Subgroup analyses will be performed based on the characteristics of physical activity intervention and population included in the studies. Ethics and dissemination This systematic review will synthesise evidence on the association of physical activity and $\mathrm{HbA} 1 \mathrm{C}$ in non-diabetic populations. This study is important from the clinical and public health point because it will estimate the effect of physical activity on the glycemic control, and it will also examine which is the type of physical activity that should be recommended for preventing type 2 diabetes and its complications. The results will be disseminated by publication in a peerreviewed journal. Ethical approval will not be required because the data used for this systematic review will be obtained from published studies and there will be no concerns about privacy.

Trial registration number PROSPERO CRD42016050991.
Strengths and limitations of this study

- This study presents a comprehensive methodology for analysing the effect of physical activity interventions on glycaemic control measured using $\mathrm{HbA1c}$ levels in general and non-diabetic populations.

- Two researchers will independently perform study selection, data extraction and quality assessment.

- The assessment of risk of bias of the selected studies and heterogeneity among studies included, with particular reference to study design and sample characteristics, is a featured point in this evidence review.

- The differences among physical activity interventions might be a source of variable quality and heterogeneity among studies, and may limit the quality of the evidence of this meta-analysis.

\section{INTRODUCTION}

Guidelines from the American Diabetes Association $^{1}$ and the $\mathrm{WHO}^{2}$ propose glycated haemoglobin A1c (HbA1c) levels of $>6.5 \%$ (48.0 $\mathrm{mmol} / \mathrm{mol})$ for the diagnosis of diabetes. Also, recent meta-analyses have reported an increase in all-cause mortality with HbA1c levels around 5.7\% (39.0 $\mathrm{mmol} / \mathrm{mol})$ in non-diabetic subjects and around $7.5 \%$ $(58.0 \mathrm{mmol} / \mathrm{mol})$ in diabetic populations. ${ }^{34}$ HbA1c is a useful biochemical test for identifying people with subclinical diabetes at the onset of clinical symptoms. ${ }^{5}$ Since microvascular complications of diabetes are present in the early stages of the disease, controlling HbAlc levels should not be restricted to the diabetic population.

Substantial evidence supports the view that physical activity reduces the risk of dying prematurely owing to its positive influence on a variety of health conditions, such 
as cardiovascular disease, diabetes and other disorders of metabolism, as well as neurological diseases, sarcopenia, osteoporosis and cancer. ${ }^{67}$ The Surgeon General's Report on Physical Activity and Health ${ }^{8}$ underscores the pivotal role of physical activity in health promotion and disease prevention. It recommends that individuals should undertake 30 min of moderate physical activity on most days of the week. Research suggests that more than $60 \%$ of adults do not achieve the recommended amount of physical activity and $25 \%$ of adults are not physically active at all. Among young people, almost $50 \%$ do not regularly practice vigorous physical activity.

A previous meta-analysis showed that higher levels of physical activity (3000-4000 MET min/week) are significantly associated with a lower risk for breast cancer, colon cancer, diabetes, ischaemic heart disease and ischaemic stroke. ${ }^{9}$ In the case of diabetes, the incidence could be reduced by up to $46 \%$ by taking part in physical activity programmes $^{10}$; moreover, these programmes have been shown to improve glycaemic control and metabolic profile among both diabetic and non-diabetic populations. ${ }^{11}$ One meta-analysis concluded that structured physical activity, such as aerobic exercise, resistance training or a combination of both, is associated with $\mathrm{HbAlc}$ reductions of $0.73 \%, 0.57 \%$ and $0.51 \%$, respectively, in patients with type 2 diabetes. Also, structured exercise lasting more than $150 \mathrm{~min}$ a week was associated with $\mathrm{HbA1c}$ reductions of $0.89 \% .^{12}$ Additionally, evidence has suggested that structured physical activity could substantially reduce the incidence of type 2 diabetes. ${ }^{13-16}$

In most industrialised countries, there is an alarming increase of the incidence of type 2 diabetes in children and adolescents with low levels of physical activity. This growing incidence parallels the childhood obesity pandemic. ${ }^{17}$ A previous meta-analysis proved the effectiveness of a high-intensity physical activity intervention on reducing adiposity, and also on mitigating the risk of type 2 diabetes and its cardiovascular complications in adulthood. $^{18}$

Thus, physical activity is widely perceived to be beneficial for preventing type 2 diabetes and for controlling glycaemic levels in patients with type 2 diabetes, but evidence supporting a positive effect in the control of glycaemic levels in healthy people is weak. ${ }^{19}$ Therefore, in view of the increasing incidence of type 2 diabetes in industrialised countries, determining the effect of physical activity interventions to control HbAlc levels in non-diabetic populations is an important public health issue.

The purpose of this protocol is to provide the methodology for a review of intervention studies dealing with the effectiveness of physical activity interventions in reducing HbA1c levels in non-diabetic populations.

\section{OBJECTIVE}

This systematic review and meta-analysis protocol presents an objective and clear procedure for the extraction of information from experimental studies (randomised controlled trials (RCTs), non-randomised experimental studies and controlled pre-post studies), in which data on changes in HbAlc levels are reported as an outcome, in order to (i) estimate the effects of physical activity on glycaemic control measured by HbAlc levels in non-diabetic populations and (ii) determine which type of physical activity (based on qualitative or quantitative characteristics) has a greater positive influence on glycaemic control.

\section{METHODS AND ANALYSIS}

This systematic review and meta-analysis protocol is based on the Preferred Reporting Items for Systematic Review and Meta-Analysis Protocols (PRISMA-P) ${ }^{20}$ and the Cochrane Collaboration Handbook. ${ }^{21}$ This protocol has been previously registered in PROSPERO (registration number: CRD42016050991).

\section{Inclusion/exclusion criteria for study selection \\ Type of studies}

Randomised controlled trials, non-randomised experimental studies and controlled pre-post studies written in English, French, Portuguese, French or Spanish.

\section{Type of participants}

Studies assessing the effect, in general and non-diabetic populations, of physical activity interventions on glycaemic control measured by HbAlc levels will be selected. Studies will be selected regardless of the age of the participants included. Studies will be excluded when they include only subjects who have been diagnosed with diabetes. When more than one study provides data referring to the same sample, we will choose the one presenting the most detailed results or providing the largest sample size.

\section{Type of interventions}

Studies reporting any type of intervention consisting mainly of physical activity (endurance, resistance or alternative exercise (such as yoga or pilates)), understood as repeated bouts of exercise over time involving more than two sessions/week with a duration of at least 3 weeks, will be eligible for inclusion. Studies comparing different types of physical activity interventions or examining a specific physical activity intervention with or without a control group will be eligible for inclusion. Also, studies consisting of advice on physical activity will be included. Nevertheless, studies combining physical activity with other health interventions, such as nutritional interventions, will be excluded when data concerning the effectiveness of physical activity programmes on glycaemic control measured by HbA1c levels cannot be extracted separately.

\section{Type of outcome assessment}

Studies in which glycaemic control is an outcome measured using any of the different methods certified by the National Glycohemoglobin Standardization Program 


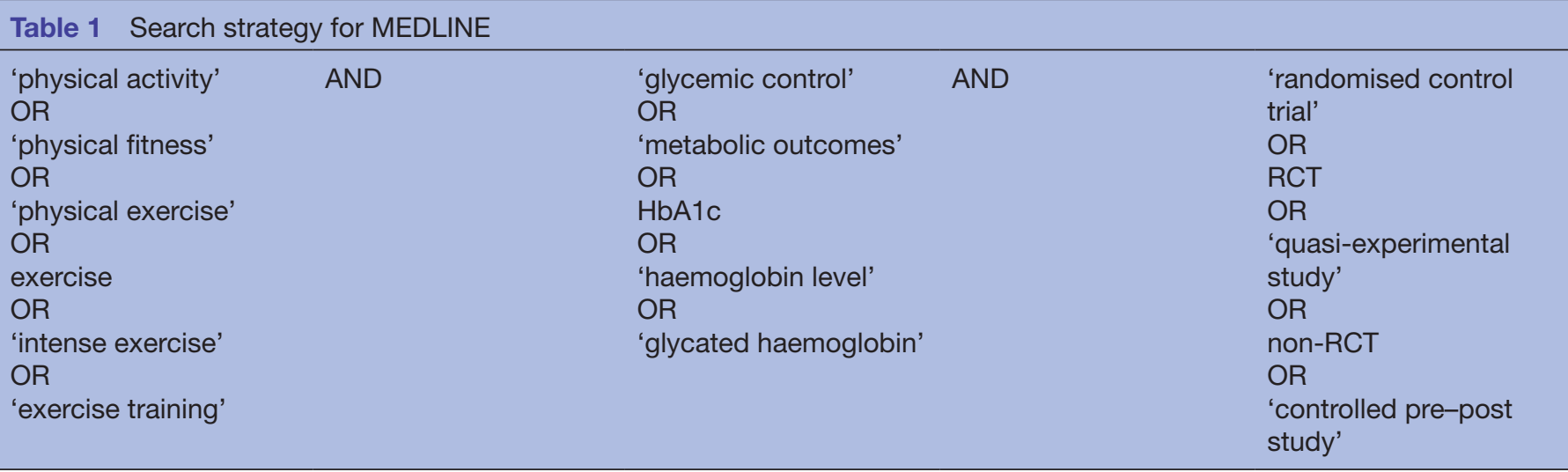

(NGSP) and standardised by the International Federation of Clinical Chemistry (IFCC) working group for testing HbA1c will be included. Studies will be included regardless of the unit in which HbAlc levels were measured-for instance, percentage $(\%)$ or $\mathrm{mmol} / \mathrm{mol}$.

\section{Search methods for the identification of studies}

\section{Electronic search}

The literature search will be conducted in MEDLINE, EMBASE, Cochrane Central Register of Controlled Trials, Cochrane Database of Systematic Reviews and Web of Science databases from inception to 31 June 2017. The searches will be re-done just before the final analyses, to search for further potential studies. Study records will be managed using the Mendeley reference manager.

The following search terms will be combined by Boolean operators for conducting the literature search: 'physical activity', 'physical fitness', 'physical exercise', exercise, 'intense exercise', 'exercise training', 'glycemic control', 'metabolic outcomes', 'HbA1c', 'haemoglobin level', 'glycated haemoglobin', 'randomised control trial', RCT, 'quasi-experimental study', non-RCT and 'controlled pre-post study' (table 1).

Previous reviews and meta-analyses, and relevant references cited in the selected studies will be screened.

\section{Data collection and analysis}

Selection of studies

The title and abstract of retrieved articles will be independently evaluated by two reviewers in order to identify eligible studies according to the inclusion criteria. Then, full manuscripts of the identified studies will be examined. Finally, the two reviewers will examine the included and excluded studies to verify the reasons for inclusion/ exclusion (figure 1). Abstracts not providing enough information regarding the inclusion/exclusion criteria will be selected for full-text evaluation. The reviewers will not be blinded to the authors, institutions or journals of the reviewed papers. Disagreements will be solved by consensus; when disagreements persist after discussion, a third reviewer will be required.

Two authors will independently extract information about the main study characteristics from the included studies including, author, year of publication, country, study design, number and age of participants, population characteristics (healthy or with any specific disease), prevalence of diabetes, methods certified by the NGSP and standardised by the IFCC used for HbAlc testing, HbA1c mean values before the intervention, and type and characteristics of the physical activity intervention (table 2). To avoid double counting of patients because they have been included in more than one report by the same author or working group, the recruitment periods will be evaluated. When necessary, corresponding authors of the potentially included studies will be contacted to obtain any missing information.

Any disagreements will be resolved by discussion to reach a consensus.

\section{Assessment of risk of bias in the included studies}

Two researchers will independently conduct a quality assessment according to the Cochrane Collaboration Handbook recomendations. ${ }^{21}$ Any disagreements will be resolved by discussion and a third reviewer will solve disagreements if consensus is not reached.

The methodological quality of the RCTs will be assessed using the Cochrane Collaboration's tool for assessing risk of bias. ${ }^{22}$ This tool evaluates the risk of bias according to six domains: selection bias, performance bias, detection bias, attrition bias, reporting bias and other bias.

The Quality Assessment Tool for Quantitative Studies ${ }^{23}$ assesses the quality of pre-post studies and non-RCTs. This tool evaluates seven domains: selection bias, study design, confounders, blinding, data collection method, withdrawals and drop-outs.

In both quality assessment tools, each domain will be considered as strong, moderate or weak, and studies will be classified as low risk of bias (with no weak ratings), moderate risk of bias (with one weak rating) and high risk of bias (with two or more weak ratings). The agreement rate between reviewers will be reported by calculating kappa statistics.

\section{Data synthesis}

The researchers will create ad hoc tables to summarise the characteristics of the included studies and any important questions related to the aim of this systematic review. The reviewers will determine whether a meta-analysis is 


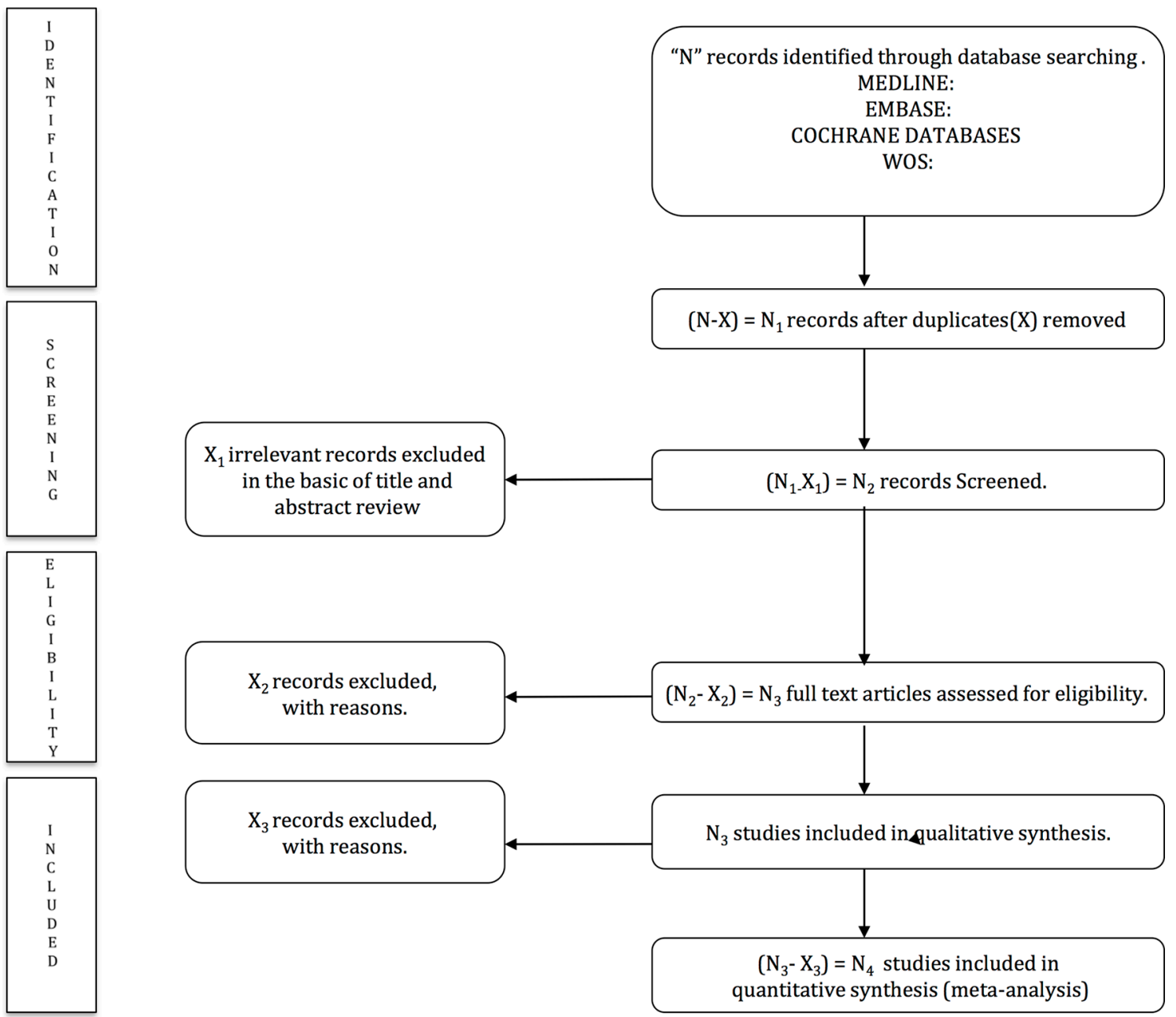

Figure 1 PRISMA flow diagram of identification, screening, eligibility and inclusion of studies.

possible after data extraction. At least five observations addressing the same specific outcome will be required to conduct a meta-analysis; where a meta-analysis is not feasible, we will undertake a narrative synthesis. Studies providing insufficient data to perform the analyses will be omitted from data syntheses.

If a meta-analysis is possible, STATA 14 software will be used to combine the pooled mean differences with $95 \%$ confidence intervals (CIs). A fixed-effects model will be used if there is no evidence of heterogeneity; otherwise, a random-effects model will be used. Study heterogeneity will be assessed with the $\mathrm{I}^{2}$ statistic. $\mathrm{I}^{2}$ values will be considered as: might not be important $(0-40 \%)$; may represent moderate heterogeneity $(30-60 \%)$; may represent substantial heterogeneity $(50-90 \%)$ and considerable heterogeneity $(75-100 \%)$, the corresponding $\mathrm{p}$ values will also be taken into account. ${ }^{21}$

Data from intention-to-treat analyses will be considered whenever available in RCTs. The HbA1c pre-post intervention mean difference will be the primary indicator of the intervention outcome. Standardised mean differences will be calculated for HbA1c levels. Finally, publication bias will be assessed using a contour-enhanced funnel plot of each effect size against the SE.
Funnel plot asymmetry will be visually evaluated, and by the method proposed by Egger, ${ }^{24}$ and significant publication bias will be considered to be present if the $p$ value is $<0.10{ }^{25}$ The trim-and-fill computation will be used to assess the effect of publication bias on the interpretation of results. ${ }^{26}$

\section{Subgroup analysis and meta-regression}

Subgroup analyses and meta-regression will be conducted by age of participants (children and/or adolescents, young adults aged 18-35 years, middle-aged adults aged $36-55$ years or older adults aged above 55 years), type of physical activity intervention (leisure-time physical activity, active commuting, physical activity programme or physical activity counselling), type of exercise (endurance, resistance or alternative exercises), length of physical activity intervention (above or below 12 weeks), physical activity duration per week (above or below $150 \mathrm{~min}$ ), type of study design (RCT, non-RCT and controlled pre-post studies), because these may be the potential major factors to cause heterogeneity. Furthermore, the methodological quality of studies included will be considered for additional subgroup analyses. 


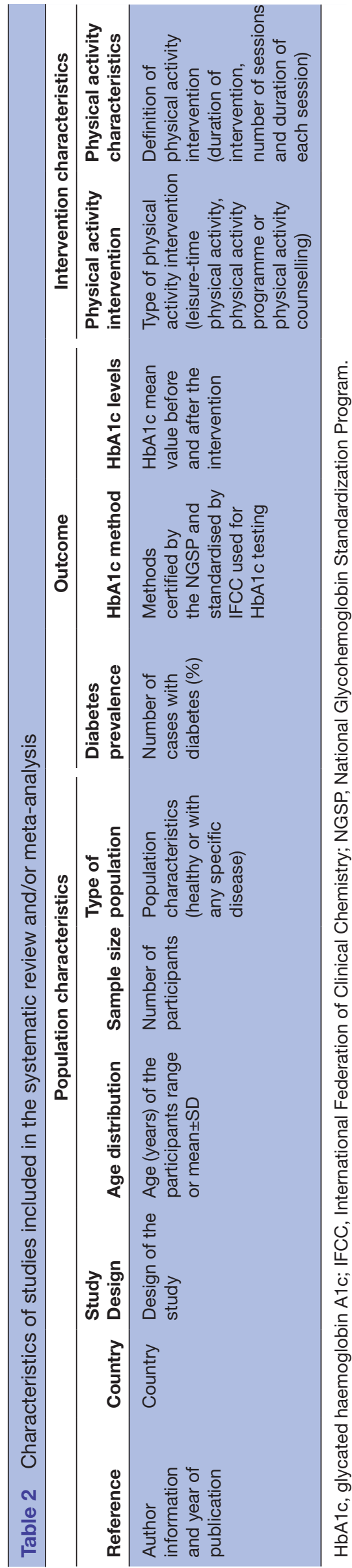

Sensitivity analysis

Sensitivity analyses will be conducted, excluding studies from the analysis one by one. These will be performed to prove that the findings from the meta-analysis do not depend on arbitrary or unclear decisions.

\section{ETHICS AND DISSEMINATION}

An association between physical activity interventions and glycaemic control measured by HbA1c levels has been reported by recent systematic reviews and meta-analyses in both type $2^{27-31}$ and type 1 diabetic populations. ${ }^{28}$ 293233 One meta-analysis ${ }^{27}$ reported no significant benefits of glycaemic control in non-diabetic populations, but included only three intervention studies divided into two subgroups (healthy and chronic disease). No previous systematic review or meta-analysis has included studies in non-diabetic subjects. Therefore, the aim of this protocol is to present a clear and reliable methodology to estimate the effects of physical activity on glycaemic control measured by HbAlc levels in non-diabetic populations.

There are some sources of heterogeneity that will be controlled in this systematic review and meta-analysis. Sources of variability will be determined by analysing the design (type of study, type of intervention and control group, sample size and length of intervention) and the sample characteristics (type of population, age range and gender distribution) of the studies included.

As different study designs will be considered for inclusion, we will use two quality assessment tools: the Cochrane Collaboration's tool for assessing risk of bias ${ }^{22}$ and the Quality Assessment Tool for Quantitative Studies. ${ }^{23}$ Both tools were rigorously developed, and are evidence-based, valid, reliable and easy to use. ${ }^{34}$

Random-effects meta-regression will be used to evaluate whether the relationship between physical activity and glycaemic levels could differ according to certain sample characteristics and whether those characteristics could be considered major sources of heterogeneity. ${ }^{35}$ Additionally, subgroup analyses in this meta-analysis will be conducted to control for heterogeneity between the studies. To determine the level of heterogeneity, we will use the definition suggested by the Cochrane Collaboration Handbook. ${ }^{21}$

Therefore, some aspects of physical activity that currently seem to be controversial will be deeply studied in this meta-analysis, such as the effect that each type of physical activity could produce on glycaemic control measured by HbA1c in non-diabetic populations. The evidence of the effect of each type of physical activity might help to establish physical activity programmes tailored to the characteristics of each subject and the proposed objectives. Moreover, whether physical activity counselling interventions that involve written advice by a health professional can increase the daily amount of time that patients spend on physical exercise-related activities should be clarified. ${ }^{36}$ Finally, another important issue to take into account in this meta-analysis will be whether 
complying with the Surgeon General's Report on Physical Activity and Health recommendations has beneficial effects on glycaemic control in non-diabetic populations.

If the study confirms the positive effects of physical activity on controlling or decreasing HbAlc levels in a non-diabetic population, then promoting physical activity should be a useful strategy to prevent diabetes mellitus, and also its micro- and macrovascular complications such as retinopathy, nephropathy, arterial stiffness or cardiovascular diseases. Thus, synthesising the evidence for the effectiveness of different types of physical activity on HbA1c levels might provide support for the inclusion of physical activity in population-based prevention interventions in different population groups (ie, children, adults, elderly). This study would also demonstrate the weaknesses of the available evidence supporting the relationship between HbAlc levels and glycaemic-related disorders, and therefore could suggest future research areas.

Potential limitations of this research may be publication bias, information bias, poor statistical analyses and inadequate reporting of methods and findings of the studies included. ${ }^{25}$ However, it is important to summarise the information available on this issue. To overcome these limitations, we will follow the recommendations included in the PRISMA ${ }^{37}$ and the Cochrane Collaboration Handbook. ${ }^{21}$

Numerous meta-analyses synthesising the effects of physical activity on glycaemic control measured by HbA1c levels in diabetic populations have already been conducted. However, there is no meta-analysis in non-diabetic populations relating physical activity with glycaemic control measured by HbAlc levels, despite the increasing number of intervention studies on this association. Therefore, it seems necessary to conduct a systematic review that may provide a global overview of the current literature and could also improve future research on this topic. This protocol provides a clear and structured procedure for maximising the extraction, and summarising the relevant information on the association of physical activity and HbAlc levels. This study will have important clinical and public health implications, because it could provide support for recommendations of physical exercise in non-diabetic subjects, which might help to prevent type 2 diabetes and its complications. According to the findings of this systematic review and meta-analysis, suggestions for future research will be made, and recommendations for evidence-based physical activity interventions for glycaemic control and prevention of diabetes mellitus in healthy subjects will be implemented.

Contributors VM-V and IC-R designed the study. VM-V was the principal investigator and guarantor. IC-R and VM-V were the main coordinators of the study. $\mathrm{BP}, \mathrm{CA}-\mathrm{B}$ and VM-V conducted the study. MG-M, BP, EA and CA-B gave statistical and epidemiological support. IC-R wrote the article with the support of EA and BP. All authors revised and approved the final version of the manuscript.

Funding This research received no specific grant from any funding agency in the public, commercial or not for profit sectors. IC-R is supported by a grant from the Universidad de Castilla-La Mancha (FPU13/01582). BP is supported by a grant from the Portuguese Foundation for Science and Technology (SFRH/BPD/108751/2015). CA-B and MG-M are supported by a grant from the Spanish Ministry of Ministry of Education, Culture and Sport (FPU13/03137 and FPU15/03847, respectively).

Competing interests None declared.

Patient consent Not applicable.

Provenance and peer review Not commissioned; externally peer reviewed. Data sharing statement Not applicable.

Open Access This is an Open Access article distributed in accordance with the Creative Commons Attribution Non Commercial (CC BY-NC 4.0) license, which permits others to distribute, remix, adapt, build upon this work non-commercially, and license their derivative works on different terms, provided the original work is properly cited and the use is non-commercial. See: http://creativecommons.org/ licenses/by-nc/4.0/

(c) Article author(s) (or their employer(s) unless otherwise stated in the text of the article) 2017. All rights reserved. No commercial use is permitted unless otherwise expressly granted.

\section{REFERENCES}

1. American Diabetes Association. (2) Classification and diagnosis of diabetes. Diabetes Care 2015;38(Supplement 1):S8-16.

2. World Health Organization. Use of glycated haemoglobin (HbA1c) in the diagnosis of diabetes mellitus: abbreviated report of a WHO consultation. Geneva: WHO, 2011.

3. Arnold LW, Wang Z. The HbA1c and all-cause mortality relationship in patients with type 2 diabetes is $\mathrm{J}$-shaped: a meta-analysis of observational studies. Rev Diabet Stud 2014;11:138-52.

4. Zhong GC, Ye MX, Cheng JH, et al. HbA1c and risks of all-cause and cause-specific death in subjects without known diabetes: a doseresponse meta-analysis of prospective cohort studies. Sci Rep 2016;6:24071.

5. Lyons TJ, Basu A. Biomarkers in diabetes: hemoglobin A1c, vascular and tissue markers. Trans/ Res 2012;159:303-12.

6. Hardman AE, Stensel DJ. Physical activity and health: the evidence explained. Routledge, 2009.

7. World Health Organization. Global recommendations on physical activity for health. Geneva: WHO, 2010.

8. US Department of Health and Human Services. Physical activity and health: a report of the surgeon general. US Department of Health and Human Services, Centers for Disease Control and Prevention, National Center for Chronic Disease Prevention and Health Promotion: Atlanta GA, 1996.

9. Kyu HH, Bachman VF, Alexander LT, et al. Physical activity and risk of breast cancer, colon cancer, diabetes, ischemic heart disease, and ischemic stroke events: systematic review and dose-response meta-analysis for the global burden of disease study 2013. BMJ 2016;354:i3857.

10. Li G, Zhang P, Wang J, et al. The long-term effect of lifestyle interventions to prevent diabetes in the China da Qing Diabetes Prevention Study: a 20-year follow-up study. The Lancet 2008;371:1783-9.

11. Festa A, Williams $K$, D'Agostino $R$, et al. The insulin resistance atherosclerosis study. Diabetes 2006;55:1114-20.

12. Umpierre D, Ribeiro PA, Krame CK, et al. Physical activity advice only or structured exercise training and association with $\mathrm{HbA} 1 \mathrm{c}$ levels in type 2 diabetes: a systematic review and meta-analysis. JAMA 2011;305:1790-9.

13. Helmrich SP, Ragland DR, Leung RW, et al. Physical activity and reduced occurrence of non-insulin-dependent diabetes mellitus. $N$ Engl J Med Overseas Ed 1991;325:147-52.

14. Lynch $\mathrm{J}$, et al. Moderately intense physical activities and high levels of cardiorespiratory fitness reduce the risk of non-insulindependent diabetes mellitus in middle-aged men. Arch Intern Med 1996;156:1307-14.

15. Fb H, Sigal RJ, Rich-Edwards JW, et al. Walking compared with vigorous physical activity and risk of type 2 diabetes in women: a prospective study. JAMA 1999;282:1433-9.

16. Laaksonen DE, Lindstrom J, Lakka TA, et al. Physical activity in the prevention of type 2 diabetes: the Finnish Diabetes Prevention Study. Diabetes 2005;54:158.65.

17. Biddle SJH, Gorely T, Stensel DJ. Health-enhancing physical activity and sedentary behaviour in children and adolescents. J Sports Sci 2004:22:679-701.

18. Garcia-Hermoso A, Cerrillo-Urbina AJ, Herrera-Valenzuela T, et al. Is high-intensity interval training more effective on improving 
cardiometabolic risk and aerobic capacity than other forms of exercise in overweight and obese youth? A meta-analysis. Obes Rev 2016;17:531-40.

19. Gay JL, Buchner DM, Schmidt MD. Dose?response association of physical activity with $\mathrm{HbA1c}$ : intensity and bout length. Prev Med 2016;86:58-63.

20. Moher D, Shamseer L, Clarke M, et al. Preferred reporting items for systematic review and meta-analysis protocols (PRISMA-P) 2015 statement. Syst Rev 2015;4:1.

21. The Cochrane Collaboration. In: Higgins JP, Green S, eds. Cochrane handbook for systematic reviews of interventions. 5.1.0. 2011. http:/ handbook.cochrane.org. (updated March 2011).

22. Higgins JPT, Altman DG, Gotzsche PC, et al. The Cochrane Collaboration's tool for assessing risk of bias in randomised trials. BMJ 2011:343:d5928.

23. National Collaborating Centre for Methods and Tools. Quality assessment tool for quantitative studies. Hamilton, ON: McMaster University, 2008. (Updated 13 April, 2010).

24. Sterne JAC, Egger M, Smith GD. Systematic reviews in health care: investigating and dealing with publication and other biases in metaanalysis. BMJ 2001;323:101-5.

25. Egger M, Smith GD, Schneider M, et al. Bias in meta-analysis detected by a simple, graphical test. BMJ 1997;315:629-34.

26. Duval S, Tweedie R. Trim and fill: a simple funnel-plot-based method of testing and adjusting for publication bias in meta-analysis. Biometrics 2000:56:455-63.

27. Byrne H, Caulfield B, De Vito G. Effects of self-directed exercise programmes on individuals with type 2 diabetes mellitus: a systematic review evaluating their effect on $\mathrm{HbA} 1 \mathrm{c}$ and other metabolic outcomes, physical characteristics, cardiorespiratory fitness and functional outcomes. Sports Med 2017;47:717-33.

28. Pai L-W, Li T-C, Hwu Y-J, et al. The effectiveness of regular leisuretime physical activities on long-term glycemic control in people with type 2 diabetes: a systematic review and meta-analysis. Diabetes Res Clin Pract 2016;113:77-85.

29. Ishiguro $\mathrm{H}$, Kodama $\mathrm{S}$, Horikawa $\mathrm{C}$, et al. In search of the ideal resistance training program to improve glycemic control and its indication for patients with type 2 diabetes mellitus: a systematic review and meta-analysis. Sports Medicine 2016;46:67-77.

30. Schwingshackl L, Missbach B, Dias S, et al. Impact of different training modalities on glycaemic control and blood lipids in patients with type 2 diabetes: a systematic review and network meta-analysis. Diabetologia 2014;57:1789-97.

31. Jelleyman C, Yates T, O'Donovan G, et al. The effects of highintensity interval training on glucose regulation and insulin resistance: a meta-analysis. Obes Rev 2015;16:942-61.

32. Yardley JE, Hay J, Abou-Setta AM, et al. A systematic review and meta-analysis of exercise interventions in adults with type 1 diabetes. Diabetes Res Clin Pract 2014;106:393-400.

33. Quirk H, Blake H, Tennyson R, et al. Physical activity interventions in children and young people with type 1 diabetes mellitus: a systematic review with meta-analysis. Diabet Med 2014;31:1163-73.

34. Sanderson S, Tatt ID, Higgins JP. Tools for assessing quality and susceptibility to bias in observational studies in epidemiology: a systematic review and annotated bibliography. Int J Epidemiol 2007;36:666-76.

35. Thompson SG, Sharp SJ. Explaining heterogeneity in meta-analysis: a comparison of methods. Stat Med 1999;18:2693-708.

36. NICE. A rapid review of the effectiveness of exercise referral schemes to promote physical activity in adults. London: Public Health Collaborating Centre-Physical Activity, 2006. www.nice.org. uk/nicemedia/pdf/PAExercise_Referral_Review_Final_May_2006. pdf.

37. Moher D, Liberati A, Tetzlaff J, et al. Preferred Reporting Items for Systematic reviews and Meta-Analyses: the PRISMA Statement. $J$ Clin Epidemiol 2009;62:1006-12. 